\title{
Temperature Programmed Desorption of Quench-condensed Krypton and Acetone in Air; Selective Concentration of Ultra-trace Gas Components
}

\author{
Taku T. Suzuki ${ }^{\dagger}$ and Isao SAKaguchi \\ National Institute for Materials Science, 1-1 Namiki, Tsukuba, Ibaraki 305-0044, Japan
}

\begin{abstract}
Selective concentration of ultra-trace components in air-like gases has an important application in analyzing volatile organic compounds in the gas. In the present study, we examined quench-condensation of the sample gas on a $\mathrm{ZnO}$ substrate below $50 \mathrm{~K}$ followed by temperature programmed desorption (TPD) (low temperature TPD) as a selective gas concentration technique. We studied two specific gases in the normal air; krypton as an inert gas and acetone as a reactive gas. We evaluated the relationship between the operating condition of low temperature TPD and the lowest detection limit. In the case of krypton, we observed the selective concentration by exposing at $6 \mathrm{~K}$ followed by thermal desorption at about $60 \mathrm{~K}$. On the other hand, no selectivity appeared for acetone although trace acetone was successfully concentrated. This is likely due to the solvent effect by a major component in the air, which is suggested to be water. We suggest that pre-condensation to remove the water component may improve the selectivity in the trace acetone analysis by low temperature TPD.
\end{abstract}

Keywords Temperature programmed desorption, cryogenic temperature, quench-condensation, trace gas analysis, krypton, acetone

(Received October 8, 2015; Accepted December 11, 2015; Published April 10, 2016)

\section{Introduction}

Gas condensation techniques on solid surfaces at low temperature are important in trace-level gas analysis. It is physically the socalled quench-condensation technique, but it is also often called "cryofocusing", which has been applied in particular to a sample injection of gas chromatography (GC). A number of studies has demonstrated successful application of the cryofocusing-GCmass spectrometry (MS) system to trace gas analysis, such as volatile organic compounds (VOCs) in the air and the exhaled breath. ${ }^{1-9}$

In the typical cryofocusing-GC-MS system, the cryofocusing typically takes place at the inlet of the GC column. The sample enrichment occurs by quench-condensation at sub-ambient temperature. The GC peak width becomes narrower with improved resolution and the signal-to-noise ratio as a consequence of focusing the sample gas in a narrow band by the cryofocusing technique.

The typical procedure of the cryofocusing-GC-MS measurement is quench-condensation of the sample gas at a certain low temperature followed by rapid heating. Thus, cryofocusing is usually used just for enrichment of the sample gas concentration, and gas components are separated at the GC column in cryofocusing-GC-MS. On the other hand, it is wellknown that the separation of quench-condensed gases is also possible by precise control of the desorption temperature after

$\dagger$ To whom correspondence should be addressed.

E-mail: suzuki.taku@nims.go.jp condensation. Actually, this is a basic principle of temperature programmed desorption (TPD) (or sometimes called thermal desorption spectroscopy), which is an established method to analyze the adsorption on solid surfaces.

TPD has been developed as a useful technique to study the adsorption and desorption kinetics on solid surfaces. ${ }^{10-12}$ In TPD, the substrate temperature is raised up with a constant ramping rate, and simultaneously, certain desorbed species are detected as a function of the substrate temperature. The desorbed species are typically measured by quadrupole mass spectrometer (QMS). In those TPD experiments, the spectrum sometimes exhibits a very sharp peak at the specific temperature depending on the desorption species. Such information is useful for analyzing the adsorption state of the atom and/or the molecule on surfaces, but in addition to the application to the adsorption-state analysis, those behaviors are interesting from the view point of selective concentration of trace gases. Furthermore, one may expect to separate different molecules with the same molecular mass by such a selective concentration technique. It is because the desorption temperature reflects the chemical characteristics of the condensed components. For example, this is the case for acetone and propionaldehyde, both of which have a molecular mass value of 58 and may be contained in exhaled breath. Whereas TPD has been employed in surface analysis for many years, the number of studies concerning TPD as a selective gas concentration technique is quite limited. This is probably partly because hundreds of complex organic compounds are contained in the air and the breath, ${ }^{13}$ and thus, the mere use of the TPD technique may be not sufficient to separate these compounds. In addition, TPD is 


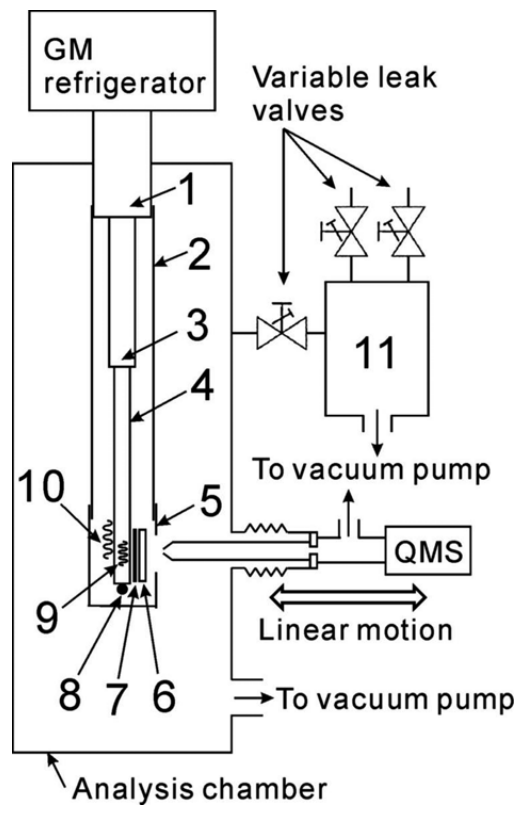

Fig. 1 A schematic illustration of low temperature TPD combined with QMS. 1, First cold head; 2, thermal shield; 3, second cold head; 4, Au plated $\mathrm{Cu}$ rod; 5, additional thermal shield; 6, $\mathrm{ZnO}$ substrate; 7 , sapphire plate; 8 , Si diode sensor; 9, tungsten filament; 10, cartridge heater; 11 , gas mixing chamber.

typically performed above room temperature, but it is necessary to cool down the substrate temperature much lower than room temperature for quench condensation of VOCs. Furthermore, azeotrope may be formed during the desorption process of TPD, which likely causes problems in the separation of compounds by TPD. Although all these are obvious disadvantages of TPD for selective gas concentration, it may have still some attractive features compared with cryofocusing-GC-MS, such as small size, the economically low cost, and easy handling.

In the present paper, we provide basic data of gas concentration by quench condensation followed by TPD (low temperature TPD) for two specific traces in the air, those are krypton and acetone. These two components were chosen in light of their inert and reactive characteristics, respectively. The motivation for the krypton analysis comes from the fact that the $\mathrm{Kr}$ concentration in air is well established to be as small as $10^{-6}$ volume fraction (ppmv) level. Thus, it is convenient to examine the performance of low temperature TPD in ultra-trace inert gas analysis. On the other hand, the acetone analysis is for the future application of low temperature TPD to exhaled breath analysis, where acetone is well-known to be a disease marker. Our present study aims to discuss the possibility of selective concentration of trace level gases in the air-like gases. The possibility, limitation, and future direction of the development of the gas concentration by low temperature TPD will be discussed in the present paper.

\section{Experimental}

Experiments were performed in a ultra-high vacuum (UHV) chamber (base pressure $7 \times 10^{-9} \mathrm{~Pa}$ ) configured for TPD using QMS with an electron multiplier option (AMETEK, Dycor) (Fig. 1). The electron multiplier option was employed for krypton and acetone, whereas it was not used for other components in the air. Thus, the ion current of QMS by the components other than krypton and acetone were measured by a conventional faraday cup. The evacuations of the UHV chamber and the QMS part were separated by an aperture with the diameter of $6 \mathrm{~mm}$. The whole set of the aperture, QMS, and the differentially pumping system was integrally retractable, and it was closely positioned to the substrate during the TPD measurement. This is to avoid the detection of the desorption species from places other than the substrate surface. The typical distance between the substrate surface and the aperture was less than $1 \mathrm{~mm}$. The ramp rate was $5 \mathrm{~K} / \mathrm{min}$ in TPD.

Our cryostat for gas condensation employed a UHVcompatible Gifford McMahon (GM) type closed-cycled He refrigerator (Iwatani, HE05) together with a homemade substrate stage and a radiation shield. The lowest temperatures around the substrate stage measured by a Si diode sensor (LakeShore, DT-670-SD-1.4H) with and without the additional radiation shield were 3.7 and $6.8 \mathrm{~K}$, respectively. The actual temperature on the substrate surface was control within $1 \mathrm{~K}$ precision. This was confirmed from our separated experiments for observing superconducting transition by electrical resistance measurements on lead $\left(T_{\mathrm{c}}=7.2 \mathrm{~K}\right)$ and niobium $\left(T_{\mathrm{c}}=9.3 \mathrm{~K}\right)$ substrates (not shown).

The substrate used for the quench condensation of gases was a mirror-polished single crystalline $\mathrm{ZnO}(0001)-\mathrm{O}$ face substrate with the size of $10 \times 10 \times 0.5 \mathrm{~mm}$. The $\mathrm{ZnO}$ substrate was electrically floated so as to enable flash heating by electron bombardment. The electrical floating was made using a sapphire plate placed with a center hole of $10 \times 10 \mathrm{~mm}$ size for the electron bombardment. The substrate was flash heated to $870 \mathrm{~K}$ for the surface cleaning before each quench-condensation. The gas condensation on the $\mathrm{ZnO}$ substrate was made by back-filling of the gas into the UHV chamber via a variable leak valve. The mixed gas of the normal air and $\mathrm{Kr}$ or acetone was prepared in the gas mixing chamber connected to the analysis chamber via the variable leak valve. The typical partial pressure of the backfilled gas was $1 \times 10^{-2} \mathrm{~Pa}$. The gas pressure measured by an ionization vacuum gauge is corrected with a gas correction factor in the present paper.

\section{Results and Discussion}

\section{Krypton}

The concentration of krypton in the ambient air is accepted as 1.14 ppmv. ${ }^{14}$ There are 6 stable isotopes in natural krypton, and ${ }^{84} \mathrm{Kr}$ is the most prevalent among those isotopes, where the natural abundance of ${ }^{84} \mathrm{Kr}$ is $0.57 .{ }^{15}$ Due to the ultra-trace abundance of ${ }^{84} \mathrm{Kr}(0.65 \mathrm{ppmv})$ in the air and its inert nature, ${ }^{84} \mathrm{Kr}$ is an appropriate ultra-trace component for evaluating the analytical instrumentation of air-like gases.

Figure 2 shows the QMS spectrum of the ambient air. The dwell time was $0.5 \mathrm{~s}$. The partial pressure of the air was $1 \times 10^{-2} \mathrm{~Pa}$, and it is almost the upper limit of the acceptable QMS working pressure according to the product specification. Thus, the measurement condition is for evaluating the lowest detection limit. Judging from the peak-to-noise ratio around the peak at $m / z$ of 84 , the lowest detection limit of the present QMS system around $\mathrm{m} / \mathrm{z}$ of 84 is estimated to be about $0.3 \mathrm{ppmv}$.

The quench condensation followed by TPD for the ambient air is shown in Fig. 3. The $\mathrm{ZnO}$ substrate was exposed to the ambient air with the pressure of $1 \times 10^{-2} \mathrm{~Pa}$ at $6 \mathrm{~K}$ for $1 \mathrm{~min}$. The rapid intensity variations of ${ }^{84} \mathrm{Kr}$ at 25 and $34 \mathrm{~K}$ coincidently occur with the desorption of major components in the air; $\mathrm{N}_{2}$ and $\mathrm{O}_{2}$ drastically desorb at those temperatures as shown in 


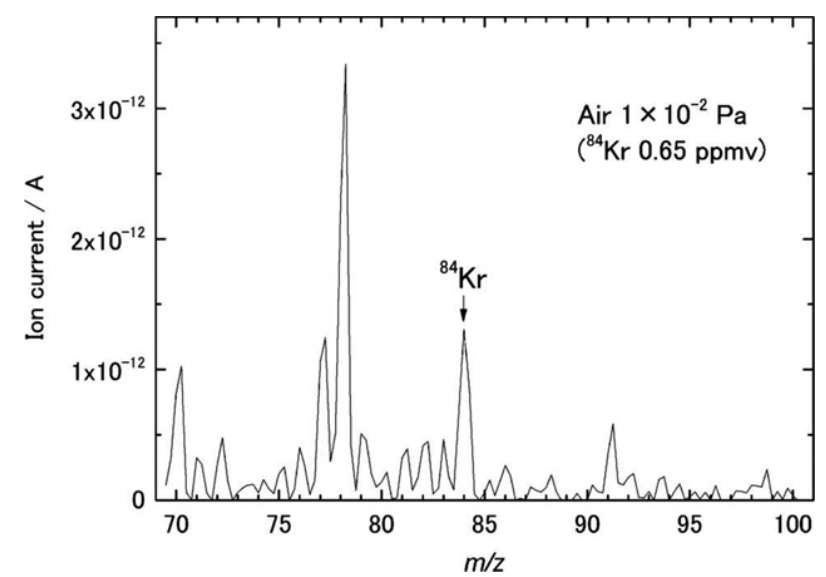

Fig. 2 QMS spectrum of the normal air with the pressure of $1 \times 10^{-2} \mathrm{~Pa}$.

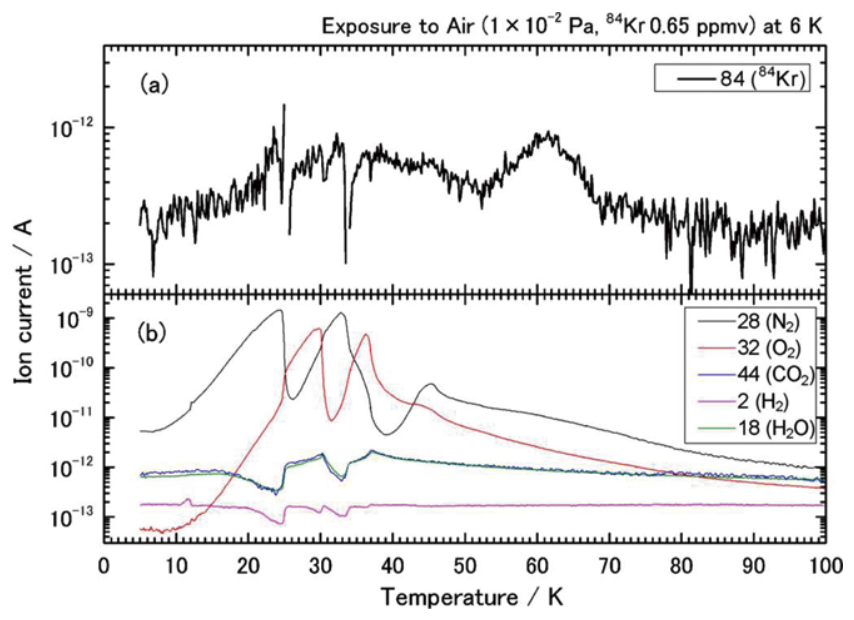

Fig. 3 TPD spectra for $m / z$ of (a) 84 and (b) 2, 18, 28, 32, and 44 . The TPD measurements were made with and without the electron multiplier in (a) and (b), respectively. The sample was prepared by the exposure of the normal air of $1 \times 10^{-2} \mathrm{~Pa}$ at $6 \mathrm{~K}$ for $1 \mathrm{~min}$.

Fig. 3(b). Thus, the ${ }^{84} \mathrm{Kr}$ peaks at 25 and $34 \mathrm{~K}$ would be induced by the desorption of those air components. Other components, such as $\mathrm{CO}_{2}, \mathrm{H}_{2}$, and $\mathrm{H}_{2} \mathrm{O}$, are also affected by the desorption of the major components of the air. On the other hand, ${ }^{84} \mathrm{Kr}$ also exhibits the peak at $61 \mathrm{~K}$, and it is independent of the intensity variation of the major component of the air in Fig. 3(b). In our separated experiments, we observed that it is also independent of $\mathrm{Ar}$ and $\mathrm{CH}_{4}$ (not shown). Thus, the desorption at $61 \mathrm{~K}$ reflects the characteristics of ${ }^{84} \mathrm{Kr}$ interaction with the substrate surface. From the viewpoint of the trace analysis, the ${ }^{84} \mathrm{Kr}$ peak at $61 \mathrm{~K}$ may be useful for selective gas concentration by low temperature TPD.

For the application of the ${ }^{84} \mathrm{Kr}$ peak at $61 \mathrm{~K}$ to the selective krypton concentration, the relationship between the ${ }^{84} \mathrm{Kr}$ peak intensity and the ${ }^{84} \mathrm{Kr}$ concentration in the air should be clarified. This is important for quantitative trace ${ }^{84} \mathrm{Kr}$ analyses by TPD. Figure 4 shows the ${ }^{84} \mathrm{Kr}$ peak intensity as a function of the ${ }^{84} \mathrm{Kr}$ concentration. The $\mathrm{ZnO}$ substrate was exposed to the mixture of the air and krypton at $6 \mathrm{~K}$ for $1 \mathrm{~min}$, where the partial pressure of the air was $1 \times 10^{-2} \mathrm{~Pa}$. The peak intensity of ${ }^{84} \mathrm{Kr}$ is estimated from the Gaussian fitting as shown in the inset of Fig. 4. The peak slightly shifts to lower temperature with the

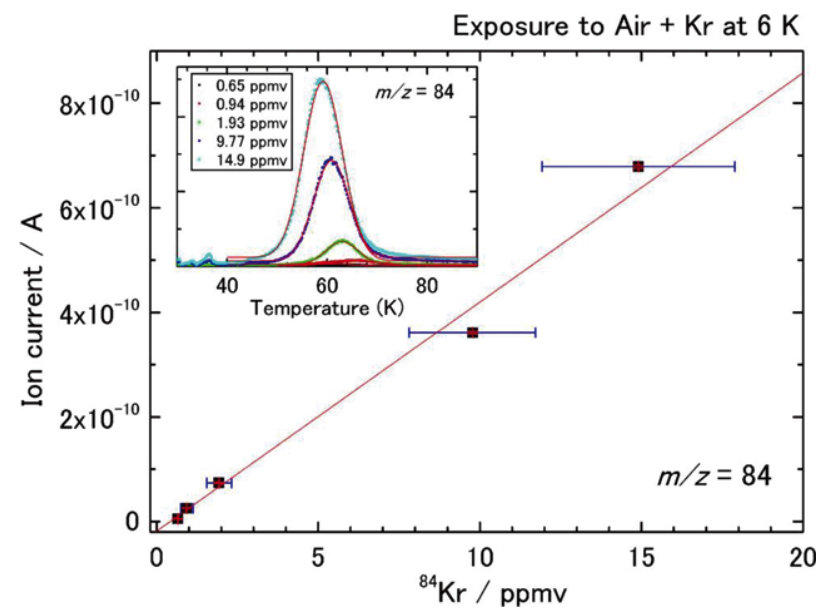

Fig. 4 TPD peak intensity at $m / z$ of 84 around $60 \mathrm{~K}$ as a function of the concentration of ${ }^{84} \mathrm{Kr}$ in the air. The inset shows TPD spectra for various concentrations of ${ }^{84} \mathrm{Kr}$ from 0.65 to $14.9 \mathrm{ppmv}$. The sample was prepared by the exposure of the normal air of $1 \times 10^{-2} \mathrm{~Pa}$ with controlled ${ }^{84} \mathrm{Kr}$ concentration at $6 \mathrm{~K}$ for $1 \mathrm{~min}$.

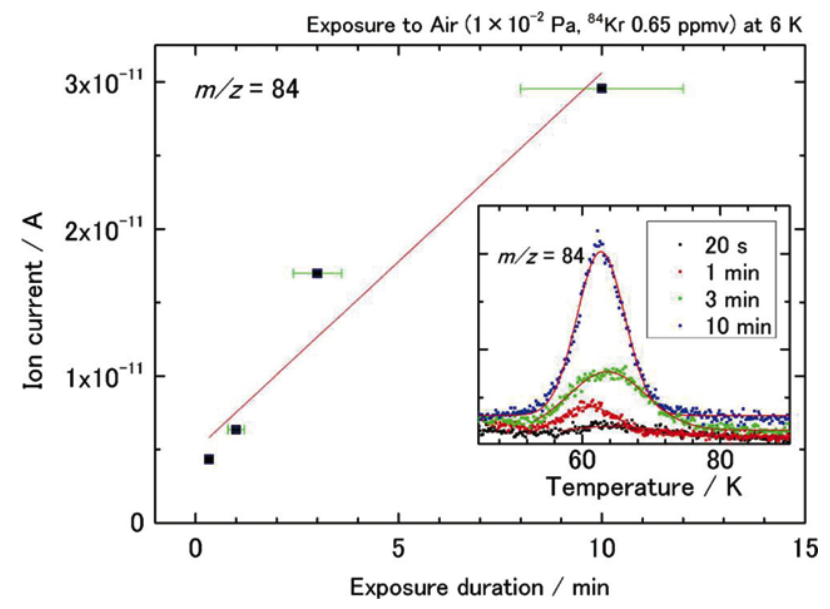

Fig. 5 TPD peak intensity at $m / z$ of 84 around $62 \mathrm{~K}$ as a function of the duration of the exposure to the normal air containing ${ }^{84} \mathrm{Kr}$ of 0.65 ppmv. The inset shows TPD spectra for various exposure durations from $20 \mathrm{~s}$ to $10 \mathrm{~min}$. The sample was prepared by the exposure of the normal air of $1 \times 10^{-2} \mathrm{~Pa}$ at $6 \mathrm{~K}$.

increase of the krypton concentration. This might be due to the enhanced interaction between krypton adsorbates in the condensed film. The linear relationship in Fig. 4 is helpful for trace krypton analysis by low temperature TPD.

It is important to elucidate the selective concentration of the trace gas component in terms of the lowest detection limit. This is demonstrated for ${ }^{84} \mathrm{Kr}$ as shown in Fig. 5. In this measurement, the $\mathrm{ZnO}$ substrate was exposed to air with the pressure of $1 \times 10^{-2}$ at $6 \mathrm{~K}$. The duration of the exposure was varied from $20 \mathrm{~s}$ to $10 \mathrm{~min}$. The lowest detection limit, which was estimated to be about 0.3 ppmv for krypton as previously mentioned, is substantially improved by the prolonged exposure. It seems that the lowest detection limit of about $0.03 \mathrm{ppmv}$ for ${ }^{84} \mathrm{Kr}$ is achieved with the exposure for $10 \mathrm{~min}$. 


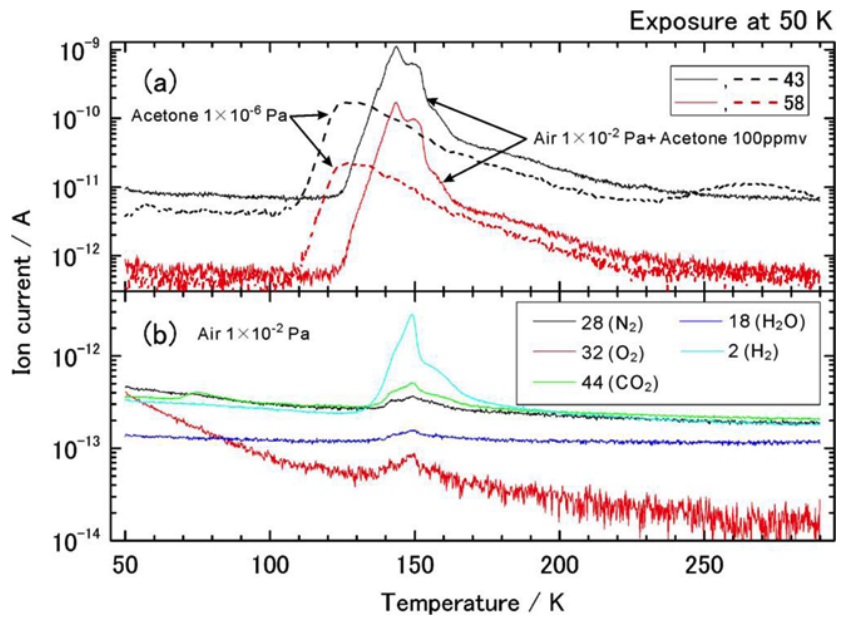

Fig. 6 TPD spectra for $m / z$ of (a) 43 and 58 and (b) 2, 18, 28, 32, and 44. The TPD measurements were made with and without the electron multiplier in (a) and (b), respectively. The sample was prepared by the exposure to the air of $1 \times 10^{-2} \mathrm{~Pa}$ (broken curves) or by the exposure to the air of $1 \times 10^{-2} \mathrm{~Pa}$ mixed with acetone of $100 \mathrm{ppmv}$ (solid curves) at $50 \mathrm{~K}$ for $1 \mathrm{~min}$.

\section{Acetone}

The acetone analysis is important for the purpose of the future application of the low temperature TPD technique to real breath analysis, where acetone is well-known to be a disease marker. ${ }^{16-21}$ We investigated low temperature TPD for acetone diluted in ambient air as shown in Fig. 6(a). The $\mathrm{ZnO}$ substrate was exposed to either the pure acetone atmosphere $\left(1 \times 10^{-6} \mathrm{~Pa}\right)$ or the mixture of air $\left(1 \times 10^{-2} \mathrm{~Pa}\right)$ and acetone $\left(1 \times 10^{-6} \mathrm{~Pa}\right)$ at $50 \mathrm{~K}$. Thus, the acetone concentration in the latter case is 100 ppmv. The coincident TPD behavior between $\mathrm{m} / \mathrm{z}$ of 43 and 58 verifies that the intensity variation in Fig. 6(a) originates from desorbed acetone and not from the fragmentation of other surface constituents. ${ }^{22}$ The desorption of the acetone occurs at different temperatures between the pure acetone and the mixture of the air and acetone. In the latter, it coincidently occurs with the major components of the air as observed in Fig. 6(b). Because the TPD peak of water is much larger than those of other components in Fig. 6(b), it is likely that the desorption of acetone diluted in the air is induced by that of water in the air. Unlike the case of ${ }^{84} \mathrm{Kr}$ in the air (Fig. 3), we observe no TPD peak of acetone which is independent of major components in the air. This is probably due to the stronger interaction of acetone with the surrounding environment (the solvent effect) compared with that of inert krypton. Thus, it is suggested that the removal of major components in the air, such as water, is critical for selective concentration of acetone in the air.

Figure 7 shows the acetone peak intensity $(\mathrm{m} / \mathrm{z}$ of 43 and 58) as a function of the acetone concentration in the air. The $\mathrm{ZnO}$ substrate was exposed to the mixture of air $\left(1 \times 10^{-2} \mathrm{~Pa}\right)$ and acetone of various partial pressure at $50 \mathrm{~K}$ for $1 \mathrm{~min}$. The acetone peak intensities correspond to the integrated ion current between 120 and $250 \mathrm{~K}$. They are fitted with a linear function, indicating that low temperature TPD is effective for the trace acetone analysis in the breath like gas. The gradient of the linear function for $\mathrm{m} / \mathrm{z}$ of 43 is about 7 times larger than that of 58. This factor resembles that in the mass spectrum database. ${ }^{22}$ It seems that the relationship between the ion current and the acetone concentration at $\mathrm{m} / \mathrm{z}$ of 43 deviates from the linear

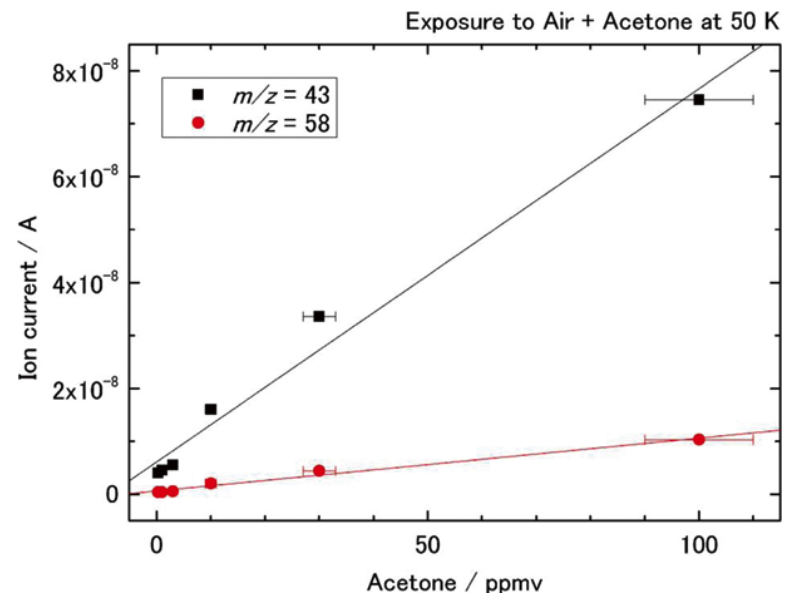

Fig. 7 TPD peak intensity at $m / z$ of 43 (black squares) and 58 (red circles) as a function of acetone concentration in the air. The sample was prepared by the exposure to the air of $1 \times 10^{-2} \mathrm{~Pa}$ with various concentrations of acetone at $50 \mathrm{~K}$ for $1 \mathrm{~min}$.

function with the increase of the acetone concentration. This may be due to the characteristic of the multiplier of our QMS. The gain of the secondary electrons in the multiplier may decrease with the ion current. This point remains unclear in the present study.

From the data in Fig. 7, the detection limit of acetone is estimated to be 0.02 and 0.14 ppmv using the TPD peak at $\mathrm{m} / \mathrm{z}$ of 43 and 58, respectively. These detection limits are estimated assuming the linearity between the ion current and the acetone concentration in the range below 3 ppmv. Moreover, it is also assumed that the resolution of the ion current in our QMS with the multiplier option is $1 \times 10^{-12} \mathrm{~A}$, which is suggested from the data in Fig. 2. These detection limits using TPD seem to be substantially improved from that of QMS $(0.2 \mathrm{ppmv})$, which is determined by the signal-to-noise ratio in the QMS spectra (Fig. 2) and electron impact ionization probability.

From the viewpoint of selective condensation of disease markers, it is desirable to remove the major component in the gas as suggested from the result shown in Fig. 6. In the case of the acetone added-air, the desorption behavior of acetone is likely determined by water as previously mentioned. Thus, if water is successfully removed from the air, the intrinsic desorption behavior of the acetone may appear, which is observed in the exposure to the pure acetone (Fig. 6(a)). The water component has been removed by using adsorbents, such as fused silica in conventional GC. ${ }^{3}$ However, careful handling of the adsorbent is necessary to avoid the contamination of sample gases. Thus, we examined the removal of the water component by pre-condensation as described below. The water component has been successfully removed by pre-condensation also in cryofocusing-GC-MS. We studied here the performance of pre-condensation in the specific case of low temperature TPD.

Thermal desorption of water with the peaks at $149 \mathrm{~K}$ occurs at a substantially higher temperature than that of acetone with the peak at $125 \mathrm{~K}$ as seen in Fig. 6. Thus, it is expected that water is dominantly removed from the acetone added-air by quench condensation between these two temperatures. Actually, this is confirmed as shown in Fig. 8. The $\mathrm{ZnO}$ substrate was exposed to air $\left(1 \times 10^{-2} \mathrm{~Pa}\right)$ with $100 \mathrm{ppmv}$ acetone at either $50 \mathrm{~K}$ (Fig. 8(a)) or $145 \mathrm{~K}$ (Fig. 8(b)) for $1 \mathrm{~min}$. In the case of the exposure experiment at $145 \mathrm{~K}$, the $\mathrm{ZnO}$ substrate was cooled to 


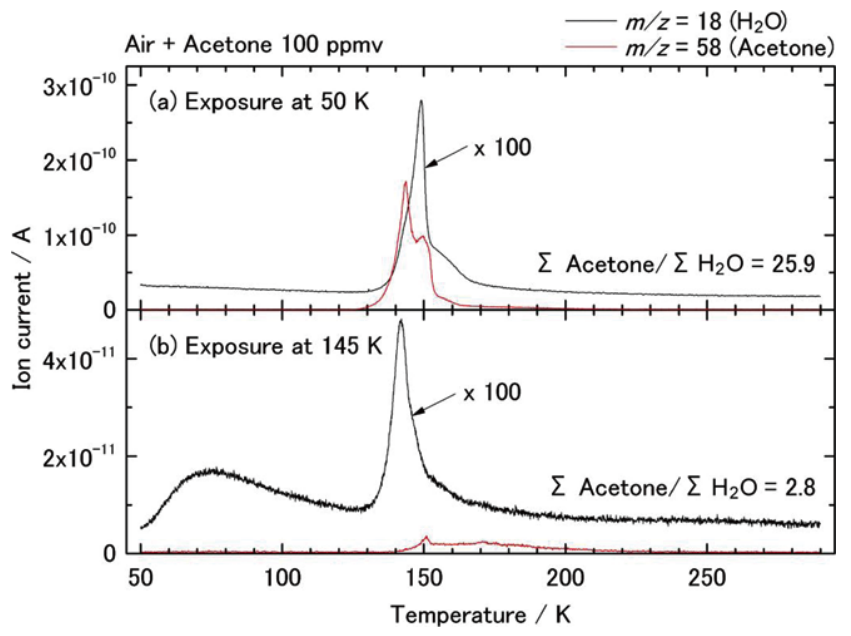

Fig. 8 TPD spectra for $m / z$ of 18 (black) and 58 (red). The TPD measurements were made with and without the electron multiplier for $\mathrm{m} / \mathrm{z}$ of 58 and 18 , respectively. The sample was prepared by the exposure to the air of $1 \times 10^{-2} \mathrm{~Pa}$ with $100 \mathrm{ppmv}$ acetone at (a) $50 \mathrm{~K}$ and (b) $145 \mathrm{~K}$.

$50 \mathrm{~K}$ after the exposure, and then TPD measurement was started. The desorption of acetone is remarkably suppressed with the exposure at $145 \mathrm{~K}$ compared with $50 \mathrm{~K}$. This is judged from the ratio of integrated ion current at $\mathrm{m} / \mathrm{z}$ of 58 (acetone) to 18 (water). Thus, the result suggests that pre-condensation at higher temperatures followed by low temperature TPD improves the trace analytical ability. The two-step quench-condensations followed by TPD may be a future direction for the development of the low temperature TPD based gas concentration method.

\section{Comparison with other gas analytical techniques}

Finally, we would like to briefly compare the low temperature TPD technique and other gas analytical methods. The present study suggests that the low temperature TPD is useful in trace gas analysis with the detection limit of sub ppmv. Although even better detection limit is expected with longer exposure duration in low temperature TPD, much better detection limit (pptv) has already been established by other recent techniques. These are the case of, for example, trace gas analysis using quantum cascade lasers ${ }^{23}$ and cavity ringdown spectroscopy (CRDS). ${ }^{24}$ These are absorption spectroscopy-based techniques, and therefore, analytes are limited by the availability of probe laser light at the appropriate wavelength. Concerning the selectivity of low temperature TPD combined with QMS, more than several different components of gases with $\mathrm{m} / \mathrm{z}$, smaller than 200 - 300 can be simultaneously measured.

Some gas analytical methods have successfully demonstrated high data throughput measurement. For example, recent CRDS studies reported a real-time response without a sample pre-process. ${ }^{24}$ This is obviously superior to low temperature TPD, which needs at least a few minutes to complete one measurement.

The size of low temperature TPD is essentially determined by a refrigerator. The present study employed a GM refrigerator with the length of about $0.5 \mathrm{~m}$ and weight of about $20 \mathrm{~kg}$. This may be smaller than some advanced cryofocus-GC-MS but larger than miniaturized GC with the detector based on metal oxide gas sensors..$^{25}$

The availability of recent advanced gas analytical techniques is often limited due to their economical cost. For example,
CRDS is rather expensive because it requires the laser system and high reflectivity mirrors. The low temperature TPD combined with QMS is not so expensive as those techniques. The main factors of expense of our setup are a refrigerator, vacuum pumps, and QMS. The refrigerator was most expensive. This is because our refrigerator can generate cryogenic temperatures such as $4 \mathrm{~K}$. The present study suggests that such a low temperature is not necessary for gas analysis by the low temperature TPD. Thus, we suppose that the expense of the low temperature TPD can be reduced in our future work.

\section{Conclusions}

We examined quench condensation of the air-like gas below $50 \mathrm{~K}$ on the $\mathrm{ZnO}$ substrate followed by TPD (low temperature TPD) as a concentration technique of trace components in gases. For the purpose of the examination with well-defined gases, we used either krypton or acetone added-ambient air. We found that low temperature TPD effectively improves the lowest detection limit of QMS for both krypton and acetone. Moreover, krypton has a TPD peak at $61 \mathrm{~K}$ that is not affected by major components in the air, and thus the selective concentration is possible for krypton by the simple low temperature TPD procedure. On the other hand, the desorption of acetone was induced by the major components of the air, which is likely water. Thus, the removal of the water component from the air is critical for the trace acetone analysis by low temperature TPD procedure. We found that the water is dominantly removed by exposing to the substrate at $145 \mathrm{~K}$. Thus, it is suggested that pre-quench condensation improves the trace analytical performance of low temperature TPD. Finally, we comment briefly on the comparison between low temperature TPD and other gas analytical methods in terms of sensitivity, selectivity, data throughput, size, and expense.

\section{Acknowledgements}

This work was partly supported by Japan Society for the Promotion of Science (JSPS) KAKENHI Grant Numbers 15H01053, 15K13366.

\section{References}

1. B. Kolb, B. Liebhardt, and L. S. Ettre, Chromatographia, 1986, 21, 305.

2. P. L. Wylie, Chromatographia, 1986, 21, 251.

3. Y. Ghoos, M. Hiele, P. Rutgeerts, and G. Vantrappen, $J$. High Resolut. Chromatogr., 1989, 12, 739.

4. S. Suzuki, Anal. Sci., 1995, 11, 953.

5. E. A. Kolbrich, R. H. Lowe, and M. A. Huestis, Clin. Chem. (Washington, DC, U. S.), 2008, 54, 379.

6. P. Tolgyessy, B. Vrana, and Z. Krascsenits, Talanta, 2011, 87, 152.

7. J. N. Smith, G. V. White II, M. I. White, R. Bernstein, and J. M. Hochrein, Am. Soc. Mass Spectrom., 2012, 23, 1579.

8. M. W. Cheong, J. Y. K. Lee, S. Q. Liu, W. Zhou, Y. Nie, E. Kleine-Benne, P. Curran, and B. Yu, Talanta, 2013, 107, 118.

9. C. Lee, Y. Lee, J. G. Lee, and A. J. Buglass, Anal. Methods, 2015, 7, 3521.

10. C. T. Campbell, Surf. Sci. Rep., 1997, 27, 1.

11. J. L. Falconer and J. A. Schwarz, Catal. Rev.-Sci. Eng., 
1983, 25, 141

12. R. J. Cvetanovr and Y. Amenomiy, Catal. Rev., 1972, 6, 21.

13. W. Cao and Y. Duan, Clin. Chem. (Washington, DC, U. S.), 2006, 52, 800 .

14. N. I. Sax, J. D. Denny, and R. R. Reeves, Anal. Chem., 1968, 40, 1915.

15. D. R. Lide, "CRC Handbook of Chemistry and Physics", 2008, CRC Press, Boca Raton, London, New York.

16. C. N. Tassopoulos, D. Barnett, and T. R. Fraser, Lancet, 1969, $1,1282$.

17. O. B. Crofford, R. E. Mallard, and R. E. Winton, Trans. Am. Clin. Climatol. Assoc., 1977, 88, 128.

18. G. Rooth and S. Ostenson, Lancet, 1970, 2, 1102.

19. M. J. Sulway and J. M. Malins, Lancet, 1970, 2, 736.

20. M. P. Kalapos, Biochim. Biophys. Acta, 2003, 122, 1621.
21. C. Deng, J. Zhang, X. Yu, W. Zhang, and X. Zhang, J. Chromatogr. B: Anal. Technol. Biomed. Life Sci., 2004, 810, 269.

22. National Institute of Standards and Technology (NIST) Chemistry WebBook, NIST Standard Reference Database Number 69, http://webbook.nist.gov/chemistry.

23. A. Kosterev, G. Wysocki, Y. Bakhirkin, S. So, R. Lewicki, M. Fraser, F. Tittle, and R. F. Curl, Appl. Phys. B: Laser Opt., 2008, 90, 165.

24. M. Sun, C. Jiang, Z. Gong, X. Zhao, Z. Chen, Z. Wang, M. Kang, Y. Li, and C. Wang, Rev. Sci. Instrum., 2015, 86, 095003

25. S. Zampolli, I. Elmi, F. Mancarella, P. Betti, E. Dalcanale, G. C. Cardinali, and M. Severi, Sens. Actuators B, 2009, 141,322 . 